\title{
Risk of brain tumours in relation to estimated RF dose from mobile phones: results from five Interphone countries
}

\author{
E Cardis, ${ }^{1}$ B K Armstrong, ${ }^{2}$ J D Bowman ${ }^{3}$ G G Giles, ${ }^{4,5}$ M Hours, ${ }^{6}$ D Krewski, \\ M McBride, ${ }^{8}$ M E Parent, ${ }^{9}$ S Sadetzki, ${ }^{10,11}$ A Woodward, ${ }^{12} \mathrm{~J}$ Brown, ${ }^{2}$ A Chetrit, ${ }^{10}$ \\ J Figuerola, ${ }^{1}$ C Hoffmann, ${ }^{11,13}$ A Jarus-Hakak, ${ }^{10}$ L Montestruq, ${ }^{6}$ L Nadon, ${ }^{9}$ \\ L Richardson, ${ }^{14}$ R Villegas, ${ }^{1} M$ Vrijheid ${ }^{1}$
}

For numbered affiliations see end of article.

\section{Correspondence to}

Professor E Cardis, Centre for Research in Environmental Epidemiology (CREAL), Hospital del Mar Research Institute (IMIM), CIBER Epidemiologia $y$ Salud Pública (CIBERESP), Doctor Aiguader 88, 08003 Barcelona, Spain; ecardis@creal.cat

Accepted 20 May 2011 Published Online First 9 June 2011

\section{UNLCK1:}

This paper is freely available online under the BMJ Journals unlocked scheme, see http:// oem.bmi.com/site/about/ unlocked.xhtml

\begin{abstract}
Objectives The objective of this study was to examine the associations of brain tumours with radio frequency (RF) fields from mobile phones.

Methods Patients with brain tumour from the Australian, Canadian, French, Israeli and New Zealand components of the Interphone Study, whose tumours were localised by neuroradiologists, were analysed. Controls were matched on age, sex and region and allocated the 'tumour location' of their matched case. Analyses included 553 glioma and 676 meningioma cases and 1762 and 1911 controls, respectively. RF dose was estimated as total cumulative specific energy (TCSE; J/kg) absorbed at the tumour's estimated centre taking into account multiple RF exposure determinants. Results ORs with ever having been a regular mobile phone user were $0.93(95 \% \mathrm{Cl} 0.73$ to 1.18$)$ for glioma and $0.80(95 \% \mathrm{Cl} 0.66$ to 0.96$)$ for meningioma. ORs for glioma were below 1 in the first four quintiles of TCSE but above 1 in the highest quintile, $1.35(95 \% \mathrm{Cl} 0.96$ to 1.90). The OR increased with increasing TCSE $7+$ years before diagnosis (p-trend 0.01; OR 1.91, 95\% Cl 1.05 to 3.47 in the highest quintile). A complementary analysis in which 44 glioma and 135 meningioma cases in the most exposed area of the brain were compared with gliomas and meningiomas located elsewhere in the brain showed increased ORs for tumours in the most exposed part of the brain in those with $10+$ years of mobile phone use (OR 2.80, 95\% Cl 1.13 to 6.94 for glioma). Patterns for meningioma were similar, but ORs were lower, many below 1.0.

Conclusions There were suggestions of an increased risk of glioma in long-term mobile phone users with high RF exposure and of similar, but apparently much smaller, increases in meningioma risk. The uncertainty of these results requires that they be replicated before a causal interpretation can be made.
\end{abstract}

\section{INTRODUCTION}

Rapid increases in mobile phone use have generated concerns about possible health effects of exposure to radio frequency (RF) electromagnetic fields. A multinational case-control study, Interphone, ${ }^{1}$ aimed to evaluate the association of brain, acoustic nerve and parotid gland tumours with RF exposure from mobile phone use. Most epidemiological studies, including Interphone, have only reported risk in relation to mobile phone use history. ${ }^{2-20}$

\section{What this paper adds}

- Previous epidemiological studies of mobile phone use and brain cancer risk have used information on mobile phone use as a proxy measure of exposure to radio frequency fields from mobile phones.

- Most studies have not observed increased ORs in relation to ever having been a mobile phone user. There were suggestions, however, of an increased risk of glioma in long-term and heavy users, though biases and errors prevent a causal interpretation.

- The relationship between radio frequency energy absorbed at the tumour location and mobile phone use history is complex. In addition to amount of use, it depends on phone type, network properties, conditions of use and tumour location. The present paper is the first to use estimates of radio frequency energy deposition at the centre of tumours in the brain as a measure of radio frequency dose.

- An increased risk of glioma was seen in individuals at the highest quintile of radio frequency dose, though reduced risks were seen in the four lower quintiles. When risk was examined as a function of dose received in different time windows before diagnosis, an increasing trend was observed with increasing radio frequency dose $(p=0.01)$ for exposures 7 years or more in the past.

- Case-case analyses, made possible by tumour localisation, indicated an increased risk in the most exposed region of the brain compared with other areas among long-term users.

- Patterns of risk for meningioma in relation to radio frequency dose were similar, although increases in risk were much smaller than for glioma, and not statistically significant.

- Our results suggest that there may be an increase in risk of glioma in the most exposed area of the brain among long-term and heavy users of mobile phones. These results are uncertain (in light of the uncertainties associated with tumour centre localisation, radio frequency dose estimation and sample size) and require replication before they can be taken to indicate a cause-effect relationship. 
The relationship between RF energy absorbed at the tumour location and mobile phone use history is complex. ${ }^{21-23}$ In addition to amount of use, it depends on phone type, network properties, conditions of use and tumour location. ${ }^{24}$ Thus, Interphone attempted a systematic and detailed evaluation and quantification of factors thought to influence RF dose from mobile phones in different brain locations. This necessitated identification of probable determinants of RF Specific Absorption Rate (SAR) during protocol development and questionnaire design and collection and analysis of information to evaluate the importance of each factor. An algorithm was developed to evaluate total RF dose absorbed at specific locations in the brain $^{24}$ and applied to Interphone study subjects in five countries to estimate RF dose at the tumour location. This paper presents the results of analysis of associations of glioma and meningioma risk with these dose estimates.

\section{MATERIALS AND METHODS}

Analyses used data from the five Interphone countriesAustralia, Canada, France, Israel and New Zealand-that agreed to transfer their data to Barcelona when EC, the Interphone Principal Investigator, relocated there. Interphone is an international, population-based case-control study based on a common protocol. ${ }^{125}$

\section{Definition of cases and controls}

Cases were patients aged 30-59 years with brain glioma or meningioma diagnosed between 2000 and 2004. Cases were ascertained actively from neurosurgical and oncological facilities, and completeness of ascertainment checked through secondary sources. ${ }^{1}$ All diagnoses were histologically confirmed or based on unequivocal diagnostic imaging.

The original Interphone protocol called for selection of one control per case from a locally appropriate population-based sampling frame. Matching variables were age (within 5 years), sex, region of residence and, in Israel, country of birth. Controls tended to be interviewed later than cases. ${ }^{1}$ As mobile phone use increased during the study period, later interviews could have spuriously increased exposure prevalence among controls. To minimise resulting bias, controls in all countries were post-hoc matched to cases with tumour localisation using an algorithm that optimised matching on interview time and age within strata defined by sex, region and, in Israel, country of birth. Date of case diagnosis was used as the reference date for cases and controls in each matched set. Each control was assigned the tumour location of his or her matched case as a reference location. To maximise statistical power, all interviewed and eligible controls were matched to glioma and meningioma cases separately, provided they had been interviewed within 1 year of the of the cases' interview; 1439 controls are thus included in both glioma and meningioma analyses. Number of controls per case varied from 1 to 19 (median 3) for glioma and 1 to 23 (median 2) for meningioma.

\section{Collection of information}

Detailed information on past mobile phone use was collected by interview with study subjects or proxies. ${ }^{1}$ This included amount, timing and conditions of mobile phone use, phone models and operators. A catalogue of phones was used to assist subjects in identifying phones they had used. In each country, historical information was obtained from mobile phone operators on communication systems and frequencies used, introduction of adaptive power control and use of discontinuous transmission.

\section{Tumour localisation}

Since intracranial RF energy deposition from mobile phones is non-uniform, with most of the energy absorbed near the phone, ${ }^{23}$ each tumour's centre was estimated so that RF dose at that location could be estimated. Neuroradiologists in each centre reviewed radiological images (MRI and CT scans) when possible and recorded tumour location and their best guess at the tumour centre (referred to as 'centre estimated by neuroradiologist') on a generic three-dimensional (3-D) grid map of the human brain, the Gridmaster, made up of $1 \mathrm{~cm}$ cubes. $^{26}$ A detailed methodological description will be published separately. $^{27}$

When images were not available, neuroradiologists used radiology reports to prepare a $3-D$ representation of the tumour volume in the Gridmaster (all cases in Israel and 12\% of cases elsewhere) (table AI). We then developed a computer algorithm to estimate tumour centre (referred to as 'centre estimated by computer algorithm'). For glioma cases, the Euclidean distances from each Gridmaster cube of the tumour's 3-D representation to the others were calculated and the cube(s) at the shortest distance(s) from others (the 'centre of gravity') chosen as the centre. For meningioma, tumours were separated into those on the surface of the meninges close to the skull and others. For the second, the process was as described for glioma; for the first, it included only cubes in the outermost Gridmaster layer. The program was validated on subjects for whom a neuroradiologist estimated the centre, with good agreement, particularly for glioma. $^{27}$

\section{RF dose algorithm}

An algorithm was developed to estimate dose as cumulative specific energy $\left(\mathrm{CSE}_{l, f s}\right)$, in joules per kilogram, absorbed at a given location in the brain (l) for a given frequency band ( $f$ ) and communication system (s) (eg, AMPS, Advanced Mobile Phone System; GSM, Global System for Mobile). ${ }^{24} \mathrm{CSE}_{l, f, s}$ was calculated as the sum, over all phones the subject used, of the product of estimated average SAR received at the tumour's location and total call duration in this frequency band and communication system. Modifying factors were taken into account: laterality of use, hands-free devices, network characteristics and, where appropriate, frequency of use in urban and rural settings. Total cumulative specific energy (TCSE) at the location was then calculated as the sum, over all frequency bands and communication systems, of the frequency- and communication-system-specific CSEs.

If the subject reported a preferred side of use, $90 \%$ of phone use was assigned to that side of the head and $10 \%$ to the other. If the subject could not answer, or said he or she used it on both sides, $50 \%$ of use was assigned to each side.

\section{Statistical analyses}

Analyses were limited to cases with tumour localisation data. The main analyses used unconditional logistic regression, stratified on the matching variables (age, sex and region) and adjusted for education level and the interaction between study region and time period of interview (6-month intervals). As the matching was on variables that were explicitly measured (and therefore could be controlled in an unconditional analysis), stratified unconditional logistic regression was conducted, rather than conditional logistic regression based on matched sets, to maximise the number of informative strata in the analyses. This was important for analyses by time windows as many matched sets would have been dropped out of these analyses if conditional logistic regression had been used. 
For the main analyses, the reference category for ORs was subjects who reported that they had never used mobile phones regularly. Sensitivity analyses restricted to regular users, with the lowest users as the reference category, were also conducted.

Dose variables included TCSE averaged over the estimated tumour centre (generally one to two cubes of the Gridmaster), TCSE averaged over the entire tumour volume and CSE for individual systems defined by frequency band (800-900 and 1800-1900 MHz) and technology type (analogue and digital). In each analysis, TCSE or CSE was censored 1 year before the reference date to allow a minimum latent period between first exposure and tumour diagnosis.

Categorical analyses were conducted in quintiles of TCSE or CSE among regular user controls. In analyses of specific systems, mobile phone users who had not used that system were assigned a CSE of 0 and included in a separate category (eg, "non $800-900 \mathrm{MHz}$ ' for analyses of $800-900 \mathrm{MHz}$ ). To allow comparisons with the 13-country Interphone analyses of mobile phone use, ${ }^{28}$ we also analysed quintiles of cumulative call time (data were too sparse for analyses by deciles).

The main analyses were based on TCSE at the tumour centre estimated by neuroradiologists when available or by computer algorithm and took into account laterality of use. We also assessed the possible effect of RF exposure in different time periods, by fitting a model in which we considered TCSE $<3$ years before the reference date, $3-6$ years before and $7+$ years before, based on reported mobile phone use in these periods. As previous studies have raised the issue of risk 10 years or more after exposure, we originally intended to analyse TCSE in periods $0-4,5-9$ and $10+$ years before the reference date but could not because of small numbers of subjects in different TCSE categories in the $10+$ years period.

Sensitivity analyses were conducted restricting analyses to subjects with probable centre estimated by a neuroradiologist; not taking into account laterality of phone use $(50 \%$ of CSE was assigned to each side for all subjects); averaging CSE over the entire tumour volume; and excluding proxy interviews, subjects with very high levels of reported phone use ( $5 \mathrm{~h}$ or more per day in any period) or subjects who reported use of $450 \mathrm{MHz}$ telephones (with little information on spatial SAR distribution, see Cardis et $\left.a l^{24}\right)$.

Finally, case-case analyses were conducted in which mobile phone use was compared between cases whose probable tumour centre was in the most exposed brain region and cases whose tumour centre was elsewhere, using unconditional logistic regression adjusted for age, sex and region. The most exposed area was defined as the area with the highest RF SAR from mobile phones, ${ }^{23}$ falling within the $3 \mathrm{~dB}$ exposure volume (figure AI), without taking into account reported laterality of use or self-reported amount of mobile phone use. It comprised 230 of the 1431 Gridmaster cubes, a region comprising about $16 \%$ of the brain volume and absorbing over $50 \%$ of the SAR from mobile phones.

\section{RESULTS}

The five Interphone countries contributing to this analysis ascertained 1302 cases of glioma, 1199 cases of meningioma and 4838 controls eligible for the study (table AI). ${ }^{1}$ Of these, 809 glioma cases and 842 meningioma cases were interviewed and could be matched to at least one control (table AI). Information on tumour localisation, communication systems and frequency bands used was available for 553 glioma cases (42.4\% of glioma cases) and 1762 matched controls (36\% of controls), and 676 meningioma cases $(56.4 \%$ of meningioma cases) and 1911 matched controls (39.5\% of controls); these were the subjects of the primary analyses of RF exposure. The mean age of glioma cases was 47.2 years and $62 \%$ were men; the mean age of meningioma cases was 49.7 years and $26 \%$ were men. The respective matched controls had similar age and sex distributions.

We calculated ORs for regular phone use and cumulative call time without use of hands-free devices in all interviewed subjects who could be matched to relate results of this fivecountry analysis to those of Interphone as a whole. ${ }^{28}$ For glioma, the OR for regular phone use was 0.92 (95\% CI 0.75 to 1.13) and that for the top category of cumulative call time (735+ h of use) was 1.17 (95\% CI 0.88 to 1.56$)$ (table 1$)$. The most closely corresponding Interphone results were, respectively, 0.81 (95\% CI 0.70 to 0.94 ) and, for 735-1639.9 and $1640+\mathrm{h}$ of use together, 1.00 (95\% CI 0.80 to 1.22 ). For meningioma, the OR for regular phone use was 0.80 (95\% CI 0.66 to 0.96$)$ and that for $735+$ h of use was 1.01 (95\% CI 0.75 to 1.36) (table 1) compared with Interphone results of, respectively, 0.79 (95\% CI 0.68 to 0.91 ) and, for $735-1639.9$ and $1640+\mathrm{h}$ of use together, 0.93 (95\% CI 0.73 to 1.19). Results of similar analyses in subjects with localisation data were not greatly different from those in all interviewed subjects (table 1). ORs for glioma in subjects whose tumour centre was also estimated by a neuroradiologist were higher-1.06 (95\% CI 0.77 to 1.47$)$ in regular users and 1.72 (95\% CI 1.07 to 2.77 ) in $735+$ h of call time, mainly due to exclusion of subjects from Israel. Israel contributed $73 \%$ of excluded glioma cases and its ORs for glioma were 0.67 (95\% CI 0.43 to 1.05$)$ in regular users and 0.57 (95\% CI 0.30 to 1.74$)$ in $735+h$ of call time.

TCSE was highly skewed in cases and controls (figure AII). Higher proportions of glioma cases than controls had TCSE above $3500 \mathrm{~J} / \mathrm{kg}$. Though there was moderate agreement between categorisation of subjects by TCSE and cumulative call time (weighted $\kappa$ 0.68), misclassification was non-negligible, particularly at higher frequency bands. ${ }^{24}$ ORs for glioma and meningioma were below 1 in the first four quintiles of TCSE. In the highest quintile, the OR for glioma was 1.35 (95\% CI 0.96 to $1.90)$ in subjects with tumour localisation and 1.66 (95\% CI 1.03 to 2.67 ) in subjects with centre estimated by a neuroradiologist (table 2). Corresponding ORs for meningioma were 0.90 (95\% CI 0.66 to 1.24 ) and 1.01 (95\% CI 0.63 to 1.62 ).

In analyses of TCSE in different time intervals before the reference date, an increased $\mathrm{OR}$ was seen for glioma in the highest category of TCSE 7+ years before diagnosis (OR 1.91, 95\% CI 1.05 to 3.47), with an irregular but reasonably consistent trend across TCSE categories $(p=0.01)$. There was no evidence of increased risk for more recent exposures. Results for meningioma were similar, but without evidence of a trend with TCSE for exposure $7+$ years before diagnosis $(p=0.24)$ (table 3$)$.

Table 4 shows the results of sensitivity analyses for associations of brain tumours with the highest quintile of TCSE. Using tumour centre estimated by computer algorithm for all subjects had little impact on the results. The ORs for glioma and meningioma fell slightly when reported laterality was not taken into account. Excluding subjects with improbable reported use ( $>5 \mathrm{~h}$ /day) or with reported use of $450 \mathrm{MHz}$ phones had no effect on ORs in the highest quintile, while excluding subjects with proxy interviews increased them slightly, particularly for glioma. The OR in the highest quintile was greatest in analyses excluding non-regular users (using the lowest quintile of TCSE as the reference category-see also table AII) and analyses restricted to subjects for whom images were available for tumour localisation. 
Table 1 ORs for brain tumours with regular use of a mobile phone and cumulative mobile phone call time in all eligible subjects, subjects with tumour localisation data and subjects with tumour centre estimated by a neuroradiologist*

\begin{tabular}{|c|c|c|c|c|c|c|c|c|c|}
\hline & \multicolumn{3}{|c|}{$\begin{array}{l}\text { All subjects matched to controls or case } \\
\text { interviewed within } 1 \text { year }\end{array}$} & \multicolumn{3}{|c|}{$\begin{array}{l}\text { Subjects with tumour centre estimated by } \\
\text { a neuroradiologist or computer algorithm } \dagger\end{array}$} & \multicolumn{3}{|c|}{$\begin{array}{l}\text { Only subjects with tumour centre estimated } \\
\text { by a neuroradiologist }\end{array}$} \\
\hline & Cases & Controls & OR (95\% CI) & Cases & Controls & OR (95\% CI) & Cases & Controls & OR $(95 \% \mathrm{CI})$ \\
\hline \multicolumn{10}{|l|}{ Glioma } \\
\hline \multicolumn{10}{|l|}{ Regular use } \\
\hline Never regular user & 266 & 834 & 1.00 & 196 & 617 & 1.00 & 117 & 361 & 1.00 \\
\hline Regular user & 542 & 1629 & $0.92(0.75$ to 1.13$)$ & 355 & 1103 & $0.93(0.73$ to 1.18$)$ & 209 & 565 & $1.06(0.77$ to 1.47$)$ \\
\hline \multicolumn{10}{|c|}{ Cumulative call time without hands-free devices (h) } \\
\hline Never regular user & 266 & 834 & 1.00 & 196 & 617 & 1.00 & 117 & 361 & 1.00 \\
\hline$<13.0$ & 69 & 234 & $0.88(0.63$ to 1.24$)$ & 44 & 174 & $0.83(0.55$ to 1.26$)$ & 25 & 102 & 0.81 (0.46 to 1.42$)$ \\
\hline $13.0-60.9$ & 103 & 327 & $0.93(0.69$ to 1.25$)$ & 68 & 223 & $0.93(0.65$ to 1.32$)$ & 46 & 134 & $1.11(0.71$ to 1.75$)$ \\
\hline $61.0-199.9$ & 110 & 383 & $0.74(0.55$ to 0.99$)$ & 63 & 264 & $0.66(0.46$ to 0.96$)$ & 39 & 136 & 0.81 (0.5 to 01.33) \\
\hline $200-734.9$ & 123 & 367 & $0.94(0.71$ to 1.26$)$ & 90 & 237 & $1.07(0.76$ to 1.50$)$ & 46 & 110 & $1.03(0.64$ to 1.67$)$ \\
\hline $735+$ & 137 & 318 & $1.17(0.88$ to 1.56$)$ & 90 & 205 & $1.25(0.88$ to 1.77$)$ & 53 & 83 & 1.72 (1.07 to 2.77$)$ \\
\hline \multicolumn{10}{|l|}{ Meningioma } \\
\hline \multicolumn{10}{|l|}{ Regular use } \\
\hline Never regular user & 356 & 807 & 1.00 & 294 & 643 & 1.00 & 157 & 398 & 1.00 \\
\hline Regular user & 486 & 1541 & $0.80(0.66$ to 0.96$)$ & 381 & 1153 & 0.77 (0.63 to 0.95$)$ & 186 & 648 & 0.77 (0.58 to 1.03$)$ \\
\hline \multicolumn{10}{|c|}{ Cumulative call time without hands-free devices (h) } \\
\hline Never regular user & 356 & 807 & 1.00 & 294 & 643 & 1.00 & 157 & 398 & 1.00 \\
\hline$<13.0$ & 102 & 247 & $0.86(0.64$ to 1.15$)$ & 80 & 198 & $0.84(0.60$ to 1.16$)$ & 43 & 117 & $0.87(0.56$ to 1.34$)$ \\
\hline $13.0-60.9$ & 101 & 301 & $0.79(0.60$ to 1.04$)$ & 82 & 225 & $0.80(0.59$ to 1.10$)$ & 46 & 137 & $0.80(0.53$ to 1.22$)$ \\
\hline $61.0-199.9$ & 97 & 347 & $0.71(0.53$ to 0.94$)$ & 73 & 257 & $0.64(0.46$ to 0.89$)$ & 35 & 156 & 0.61 (0.39 to 0.96$)$ \\
\hline $200-734.9$ & 89 & 349 & $0.69(0.51$ to 0.93$)$ & 67 & 265 & $0.63(0.45$ to 0.88$)$ & 29 & 141 & $0.57(0.35$ to 0.94$)$ \\
\hline $735+$ & 97 & 297 & $1.01(0.75$ to 1.36$)$ & 79 & 208 & $1.06(0.75$ to 1.48$)$ & 33 & 97 & $1.30(0.79$ to 2.15$)$ \\
\hline
\end{tabular}

${ }^{*}$ Analyses based on unconditional logistic regression stratified on age, sex and region and adjusted for education and timing of interview.

†Origin is as estimated by a neuroradiologist when available or as estimated by computer algorithm otherwise.

Considering frequency band and technology type (analogue or digital) (table 4), ORs were increased somewhat for glioma in the highest quintile of CSE in the $800-900 \mathrm{MHz}$ frequency range and the highest two quintiles in the $1800-1900 \mathrm{MHz}$ range. The OR for meningioma was also elevated in the highest quintile in the $800-900 \mathrm{MHz}$ range, but much reduced in the highest quintile in the $1800-1900 \mathrm{MHz}$ range. There was little evidence of increased risk when considering analogue and digital technologies separately, except possibly for analogue signals with meningioma. These analyses are not independent, however, as many subjects had used several different frequency bands or technologies. Data were too sparse for meaningful analyses of subjects who only used one frequency band or system. ORs for glioma were above 1 in all countries except Israel; CIs were wide; however, the $\mathrm{p}$ value for heterogeneity across countries was 0.37 . Country-specific ORs for meningioma were more variable. There was little evidence of differences in ORs between men and women.

For the case-case analyses, there were 44 glioma cases and 135 meningioma cases located in the most exposed area of the brain and 512 and 537 cases, respectively, located elsewhere. Knowledge of SAR was used to define the two case groups, thus

Table 2 ORs for brain tumours with level of total cumulative specific radio frequency energy (total cumulative specific energy) (in joules per kilogram)*

\begin{tabular}{|c|c|c|c|c|c|c|}
\hline & \multicolumn{3}{|c|}{$\begin{array}{l}\text { Subjects with tumour centre estimated by } \\
\text { a neuroradiologist or computer algorithm } \dagger\end{array}$} & \multicolumn{3}{|c|}{$\begin{array}{l}\text { Only subjects with tumour centre estimated } \\
\text { by a neuroradiologist }\end{array}$} \\
\hline & Cases & Controls & OR $(95 \%$ Cl) & Cases & Controls & OR (95\% CI) \\
\hline \multicolumn{7}{|l|}{ Glioma } \\
\hline Never regular user & 196 & 617 & 1.00 & 117 & 361 & 1.00 \\
\hline$<76.7$ & 67 & 265 & 0.76 (0.53 to 1.09 ) & 36 & 150 & 0.84 (0.51 to 1.36$)$ \\
\hline $76.7-$ & 68 & 227 & 0.94 (0.66 to 1.35$)$ & 43 & 128 & $1.00(0.62$ to 1.60$)$ \\
\hline $284.1-$ & 60 & 207 & 0.80 (0.54 to 1.18$)$ & 39 & 102 & $1.15(0.69$ to 1.90$)$ \\
\hline $978.9-$ & 57 & 197 & $0.89(0.61$ to 1.30$)$ & 34 & 99 & 0.92 (0.55 to 1.53 ) \\
\hline $3123.9+$ & 103 & 207 & $1.35(0.96$ to 1.90$)$ & 57 & 86 & 1.66 (1.03 to 2.67$)$ \\
\hline \multicolumn{7}{|l|}{ Meningioma } \\
\hline Never regular user & 294 & 643 & 1.00 & 156 & 396 & 1.00 \\
\hline$<76.7$ & 103 & 261 & 0.90 (0.67 to 1.21$)$ & 51 & 150 & 0.86 (0.57 to 1.29$)$ \\
\hline $76.7-$ & 71 & 199 & $0.74(0.53$ to 1.04$)$ & 47 & 127 & 0.95 (0.62 to 1.44$)$ \\
\hline $284.1-$ & 56 & 233 & $0.56(0.39$ to 0.80$)$ & 29 & 136 & 0.53 (0.32 to 0.87$)$ \\
\hline $978.9-$ & 62 & 209 & $0.72(0.51$ to 1.02$)$ & 23 & 117 & $0.55(0.32$ to 0.93$)$ \\
\hline $3123.9+$ & 88 & 251 & 0.90 (0.66 to 1.24$)$ & 35 & 114 & 1.01 (0.63 to 1.62 ) \\
\hline
\end{tabular}

${ }^{*}$ Analyses based on unconditional logistic regression stratified on age, sex and region and adjusted for education and timing of interview.

†Centre is as estimated by a neuroradiologist when available or as estimated by computer algorithm otherwise. 
Table 3 ORs for glioma and meningioma with level of total cumulative specific energy exposure (in joules per kilogram) in different windows of time before diagnosis of the case in subjects with probable tumour centre estimated by a neuroradiologist or computer algorithm*

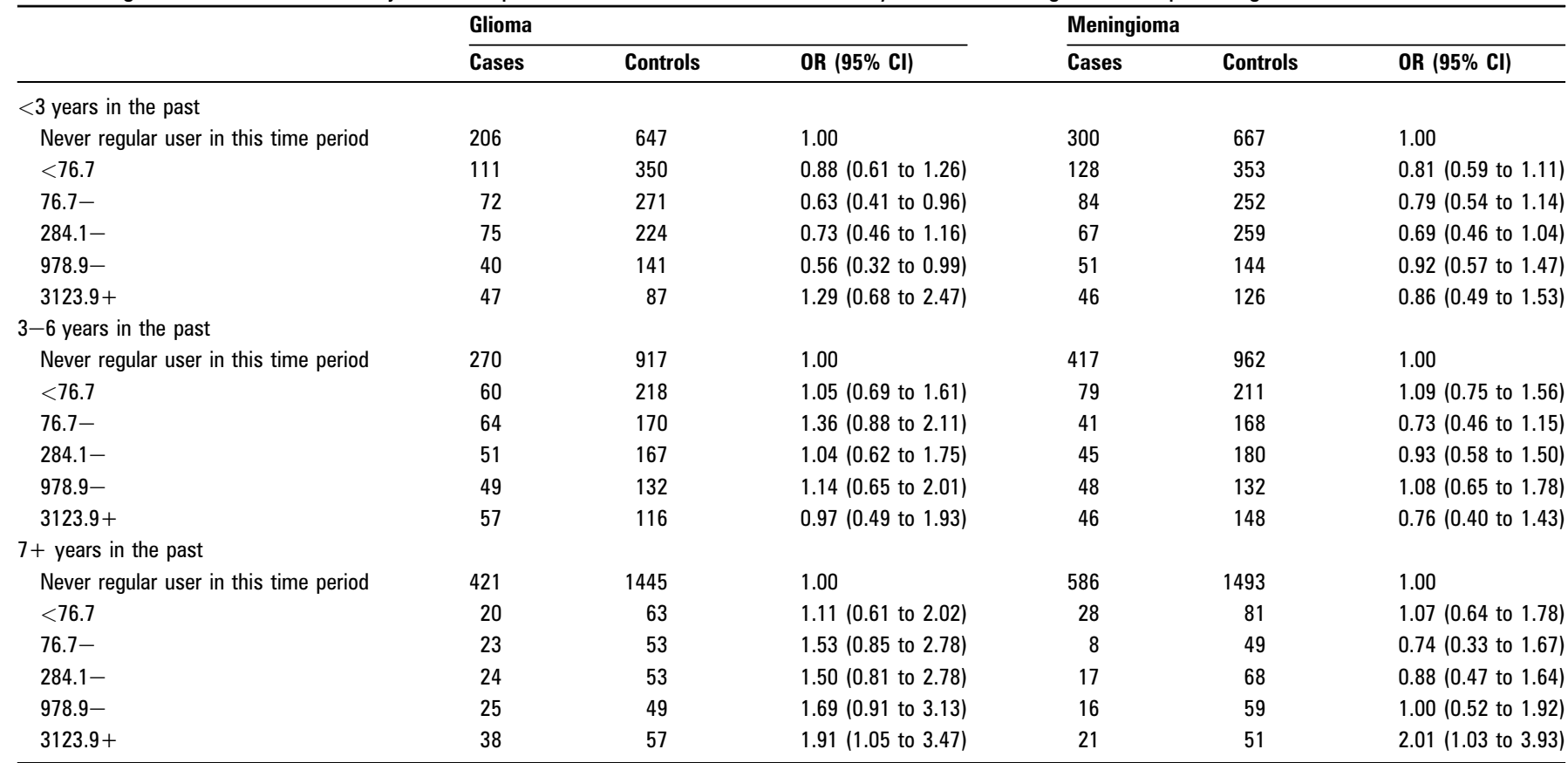

*Unconditional logistic regression analyses stratified on age, sex and region and adjusted for education and timing of interview. Centre is taken to be as estimated by a neuroradiologist where available and as estimated by computer algorithm otherwise. Associations in each time window are adjusted for associations in each other window.

only mobile phone use was compared between them. The ORs for glioma in the highest exposed area were higher in long-term users than in short-term users (OR 2.80, 95\% CI 1.13 to 6.94 for 10 years or more of use). While OR for glioma tended to increase with increasing call time to the fourth quintile, it was 0.99 in the highest quintile (table 5). For meningioma, ORs were highest in long-term and heavy users, but CIs were wide.

\section{DISCUSSION}

We have presented results of a first attempt to analyse brain tumour risk in relation to amount of RF absorbed by the brain from use of a mobile phone held to the ear. In principle, a measure of absorbed RF should be a more accurate indicator of RF exposure to neural tissue than just mobile phone use and enhance our ability to detect causal associations that might exist between exposure to mobile phone RF fields and brain tumours. Although our estimate of RF dose takes into account reported phone use (which is subject to recall bias and uncertainties ${ }^{28}$ ), it also incorporates objective parameters (location of tumour, frequency band and characteristics of communication systems), not influenced by the interviewees recall, which affect the amount of RF energy received at the tumour location. ${ }^{24}$ The localisation of tumours, necessary for accurate estimation of relevant RF absorption, made our case-case analysis by regions of the brain possible.

Overall, there was weak evidence of stronger associations of glioma and meningioma when a comprehensive estimate of RF dose rather than just mobile phone use was used in the casecontrol analysis. For glioma, the OR for the highest quintile of TCSE was 1.35; that for the highest quintile of cumulative call time was 1.25. There was a similar pattern for meningioma. There are several possible explanations for this comparative lack of increase in ORs using TCSE: observations of increased brain tumour risk with mobile phone use are due to biases, and there is no true association between mobile phone RF and brain tumours; the associations of brain tumours with TCSE are greatly weakened by sources of uncertainty in our dose estimates; or a metric other than the absorbed energy better reflects the carcinogenic mechanism. ${ }^{24}$ Furthermore, the small increases observed could be due to confounding of RF absorption with pulsed extremely low frequency (ELF) magnetic fields from digital phones. ${ }^{24}$ There is little support for this, though, when we compare ORs for analogue and digital systems (table 4) or in our case-case analyses in which the most exposed region was defined on the basis of RF absorption, which is much more localised than ELF absorption. With respect to the performance of TCSE as a dose measure, it should be noted that while cumulative call time contributed to the calculation of TCSE and there was moderate agreement between the two measures, there was important residual variation due to the inclusion of measures of RF absorption in TCSE. ${ }^{24}$ It is thus possible that investigation of different dose metrics and quantification of related uncertainties may yet improve RF dose estimation. ${ }^{24}$

The results of our case-case analysis show a moderately strong association of glioma with mobile phone use that started $10+$ years before diagnosis, OR 2.80, and little increase with shorter latency. Because of the nature of the case-case analyses, bias due to differential participation of cases and controls is ruled out; systematic differences in recall between cases with tumours in the heavily exposed region of the brain and those with tumours in the less heavily exposed region seem improbable since cases are not aware of exact tumour location and its meaning regarding exposure. The comparatively small number of subjects from five countries on which this analysis is based makes the results of the present case-case analysis much more uncertain than they would be if based on Interphone as a whole.

We also observed evidence of increased glioma risk with a long latent period after first use of a mobile phone in our standard case-control study analysis. The OR for glioma in the highest quintile of TCSE was 1.91 for exposure beginning 7+ years in the past (numbers of subjects by quintile of TCSE were too small for meaningful analyses of exposures $10+$ years in the past), and 
Table 4 Results of sensitivity analyses on ORs for glioma and meningioma with radio frequency exposure in the highest quintile of total cumulative specific energy (TCSE)

\begin{tabular}{|c|c|c|c|c|c|c|}
\hline \multirow[b]{2}{*}{ Factors included in sensitivity analyses } & \multicolumn{3}{|l|}{ Glioma } & \multicolumn{3}{|c|}{ Meningioma } \\
\hline & Cases & Controls & OR (95\% Cl) & Cases & Controls & OR (95\% Cl) \\
\hline \multicolumn{7}{|l|}{ All subjects with tumour localisation } \\
\hline \multicolumn{7}{|l|}{ TCSE at centre of tumour } \\
\hline Predicted by computer algorithm for all subjects & 99 & 206 & $1.32(0.93$ to 1.86$)$ & 93 & 253 & 0.93 (0.68 to 1.27$)$ \\
\hline $\begin{array}{l}\text { Estimated by neuroradiologists when available, predicted by computer } \\
\text { algorithm otherwise not taking account of tumour laterality }\end{array}$ & 105 & 241 & $1.23(0.89$ to 1.72$)$ & 94 & 288 & $0.84(0.62$ to 1.15$)$ \\
\hline Taking into account reported tumour laterality & 107 & 224 & $1.28(0.92$ to 1.80$)$ & 93 & 253 & $0.92(0.69$ to 1.24$)$ \\
\hline Not taking into account tumour laterality & 111 & 254 & $1.21(0.87$ to 1.68$)$ & 94 & 291 & 0.84 (0.61 to 1.15$)$ \\
\hline \multicolumn{7}{|l|}{ Effect of exclusion of } \\
\hline Subjects with very high reported use ( $>5 \mathrm{~h} /$ day) & 93 & 188 & $1.34(0.94$ to 1.91$)$ & 81 & 235 & 0.91 (0.66 to 1.26$)$ \\
\hline Subjects with proxy response & 94 & 207 & $1.43(0.99$ to 2.05$)$ & 87 & 249 & 0.93 (0.68 to 1.27$)$ \\
\hline Subjects reporting use of $450 \mathrm{MHz}$ telephones & 101 & 202 & $1.35(0.96$ to 1.91$)$ & 88 & 246 & $0.92(0.67$ to 1.26$)$ \\
\hline $1800-1900 \mathrm{MHz}$ & 22 & 57 & $1.45^{*}(0.80$ to 2.63$)$ & 6 & 59 & $0.28(0.10$ to 0.79$)$ \\
\hline \multicolumn{7}{|l|}{ By communication system } \\
\hline Analogue & 43 & 82 & $1.22(0.77$ to 1.92$)$ & 27 & 109 & $1.29(0.71$ to 2.34$)$ \\
\hline Digital & 79 & 194 & $1.22(0.84$ to 1.76$)$ & 87 & 214 & $0.87(0.45$ to 1.67$)$ \\
\hline \multicolumn{7}{|l|}{ Only subjects with centre estimated by neuroradiologists } \\
\hline Using centre estimated by neuroradiologist & 57 & 86 & $1.66(1.03$ to 2.67$)$ & 35 & 114 & 1.01 (0.63 to 1.62$)$ \\
\hline Using centre predicted by computer algorithm for all subjects & 53 & 85 & $1.58(0.98$ to 2.57$)$ & 39 & 118 & $1.02(0.64$ to 1.61$)$ \\
\hline Only subjects for whom images were available (ie, excluding Israel) & 70 & 109 & $1.82(1.20$ to 2.76$)$ & 33 & 113 & $1.23(0.76$ to 2.00$)$ \\
\hline \multicolumn{7}{|l|}{ Country-specific estimates } \\
\hline Australia & 30 & 49 & $1.15(0.56$ to 2.38$)$ & 19 & 67 & 0.75 (0.38 to 1.48$)$ \\
\hline Canada & 21 & 27 & 3.48 (1.63 to 7.43$)$ & 7 & 33 & 1.51 (0.58 to 3.93$)$ \\
\hline France & 11 & 29 & $1.39(0.56$ to 3.49$)$ & 7 & 29 & $0.77(0.30$ to 1.98$)$ \\
\hline
\end{tabular}

${ }^{*} \mathrm{OR}$ in fourth quintile: $1.86,95 \% \mathrm{Cl} 1.07$ to 3.22 (29 cases and 54 controls).

there was evidence of a trend towards increasing risk with increasing exposure. Lack of such a trend has been a consistent feature of Interphone analyses of risk of glioma and may suggest that increased ORs in the highest cumulative mobile phone use category are due to observed greater overestimation of more distant past mobile phone use by cases than controls. ${ }^{29}$ The apparent dose-response relationship for exposure 7+ years ago, however, suggests that bias is not a sufficient explanation for the increased OR in the highest TCSE category, at least in this exposure period, and suggests that the increased OR seen in the highest category of use in the Interphone report is valid. These results also suggest that if there is a real effect of RF on brain tumour risk, a combination of a minimum latent period and amount of exposure may be needed for an increased risk to be observable (it is of note that the highest category of use in this study, $735 \mathrm{~h}$ or more corresponds to about $12-13 \mathrm{~min}$ of use a day over 10 years).

A number of case-control studies, including Interphone, ${ }^{28}$ have reported stronger associations with phone use on the side of the head with the tumour. We dealt with exposure laterality in the present analysis by allocating $90 \%$ of reported phone use to the preferred side of phone use and $10 \%$ to the other side when there was a preferred side $(87 \%$ of cases and $91 \%$ of controls); otherwise, $50 \%$ was allocated to each side. In a sensitivity analysis in which we allocated $50 \%$ to each side in all subjects, the ORs for glioma and meningioma in the highest quintile of TCSE averaged over the whole tumour were only slightly reduced (table 4). Like comparisons of ORs for RF absorption with cumulative mobile phone use, this lack of marked change in OR may indicate that estimation of RF absorption has not greatly improved overall exposure ascertainment in this study. It also suggests that the increased glioma ORs are not sensitive to errors in recall of laterality of mobile phone use, which is suggested also by our casecase analyses in which laterality of use was not taken into account.

Associations observed with higher levels of TCSE exposure are much weaker for meningioma than glioma, being barely evident with TCSE in the case-control analyses except in the highest category in those first exposed $7+$ years before diagnosis (table 3). The ORs for meningioma in intermediate TCSE exposure categories are also sometimes much less than those for glioma (tables 2 and 3) and below unity. We have no certain explanation for these differences, except that they could suggest biases affecting results for both tumours (more for meningioma), a causal association of mobile phone RF with both (stronger for glioma than for meningioma) or, perhaps, a shorter latency for glioma occurrence. 
Table 5 Results of case-case analyses of glioma and meningioma by time since start of use of mobile phones and cumulative call time comparing cases* originating in the region of the brain within the $3 \mathrm{~dB}$ exposure volume ( $16 \%$ of brain volume receiving $50 \%$ of the total absorbed energy) to cases $†$ originating in other areas of the brain $\neq$

\begin{tabular}{|c|c|c|c|c|c|c|}
\hline & \multicolumn{3}{|l|}{ Glioma } & \multicolumn{3}{|l|}{ Meningioma } \\
\hline & $\begin{array}{l}\text { Centre within most } \\
\text { exposed area* }\end{array}$ & $\begin{array}{l}\text { Centre outside most } \\
\text { exposed area } \dagger\end{array}$ & OR (95\% Cl) & $\begin{array}{l}\text { Centre within most } \\
\text { exposed area* }\end{array}$ & $\begin{array}{l}\text { Centre outside most } \\
\text { exposed area } \dagger\end{array}$ & OR (95\% Cl) \\
\hline \multicolumn{7}{|l|}{ Regular user } \\
\hline Ever regular user & 30 & 334 & 1.35 (0.64 to 2.87 ) & 69 & 316 & $0.74(0.49$ to 1.11$)$ \\
\hline \multicolumn{7}{|c|}{ Time since start of use (years) } \\
\hline Never regular user & 14 & 178 & 1.00 & 66 & 221 & 1.00 \\
\hline $5-9$ & 7 & 147 & $0.72(0.27$ to 1.90$)$ & 22 & 112 & 0.75 (0.42 to 1.34$)$ \\
\hline $10+$ & 11 & 54 & 2.80 (1.13 to 6.94$)$ & 9 & 25 & 1.34 (0.55 to 3.25$)$ \\
\hline \multicolumn{7}{|c|}{ Cumulative call time without hands-free devices (h) } \\
\hline Non-regular & 14 & 178 & 1.00 & 66 & 221 & 1.00 \\
\hline$<39$ & 6 & 65 & $1.19(0.40$ to 3.51$)$ & 16 & 97 & $0.55(0.29$ to 1.02$)$ \\
\hline $39-$ & 4 & 67 & 0.93 (0.27 to 3.14$)$ & 21 & 78 & 0.93 (0.51 to 1.68$)$ \\
\hline
\end{tabular}

*Treated as 'cases'.

†Treated as 'controls'.

$¥$ Quintiles of cumulative call time among 'controls' (glioma cases with tumour centre outside the most exposed area). Centre is as estimated by a neuroradiologist when available or as estimated by computer algorithm otherwise. Unconditional logistic regression analyses were done, stratified on age, sex and region and adjusted for education and timing of interview.

A major strength of this paper is improved ascertainment of relevant exposure, which we presume to be mobile phone RF energy absorbed at the tumour location when the phone is in use near the ear. It is a weakness, though, that tumour localisation was only available for about half the cases. Small increases in strengths of associations when comparing results for TCSE with those for cumulative phone use suggest that there was a small increase in measurement accuracy (assuming a causal association) from estimation of RF dose at the tumours' centres. This suggestion is supported by the further increases in ORs when only tumours for which a neuroradiologist had estimated the centre were included in analyses. However, this analysis excluded Israeli subjects, for which the OR for any use of a mobile phone was less than that for the other four centres (OR without Israel 1.06), which weakens this inference. Furthermore, when neuroradiologists estimates of the centre were replaced by computer generated estimates, the OR for glioma in the highest category of TCSE fell only from 1.66 to 1.58 . Thus neuroradiologists' localisations of the centre at the tumour's centre of gravity may have been no more accurate than the computer algorithms.

The association of glioma with estimated TCSE seemed to be robust against subjects' recall of implausibly high mobile phone use: exclusion of those with use $>5 \mathrm{~h}$ reduced the OR minimally from 1.35 to 1.34 . In contrast, in Interphone as a whole, the reduction was from 1.40 to 1.27 . While this difference might be due to different exposure measures, it could also be due to the use of a subset of Interphone countries in this analysis.

Generally speaking, results of our analyses are similar to comparable analyses based on all Interphone centres. ${ }^{28}$ One other study, from Sweden, has investigated associations of mobile phone use with brain tumour risk based on tumours diagnosed over a period, 2000 to 2003, nearly the same as Interphone, 2000 to $2004 .^{9}$ That study found increased risks of malignant brain tumours (mostly gliomas) with any use of analogue or digital mobile phones or cordless phones; risk was generally increased in both low- and high-exposure categories, evident with exposure $1-5$ years before diagnosis and increasing with intensity of exposure in time intervals more distant from diagnosis. Our results are different from this study's results with respect to the apparent limitation of an association between exposure and glioma in our data to higher exposures and exposures occurring $7+$ or $10+$ years before diagnosis.

Our use of estimates of RF energy absorbed in the brain as indicators of dose in analyses of associations of brain tumour occurrence with mobile phone use may have increased the strength of some positive associations otherwise observable between these tumours and estimated cumulative use of a mobile phone. Although subject to considerable uncertainty, our analyses suggest that there is an increase in glioma risk with higher levels of RF dose in people whose brain has absorbed high levels of RF energy from mobile phone use and that this risk may only be evident in people who began mobile phone use 7-10 years or more before diagnosis. There is a possibility also of similar, but apparently much smaller, increases in meningioma risk. Uncertainties around these results require that they are replicated before they can be considered to be real. The best way to replicate them would be to repeat this analysis in data from the other eight Interphone countries and in future studies with longer latency periods and higher cumulative exposures.

\section{Author affiliations}

${ }^{1}$ Centre for Research in Environmental Epidemiology (CREAL), Hospital del Mar Research Institute (IMIM), CIBER Epidemiologia y Salud Pública (CIBERESP), Barcelona, Spain

${ }^{2}$ Sydney School of Public Health, The University of Sydney, Sydney, Australia ${ }^{3}$ Engineering and Physical Hazards Branch, National Institute for Occupational Safety and Health (NIOSH), Cincinnati, Ohio, USA

${ }^{4}$ Cancer Epidemiology Centre, Cancer Council Victoria, Melbourne, Australia ${ }^{5}$ Centre for MEGA Epidemiology, School of Population Health, The University of Melbourne, Melbourne, Australia

${ }^{6}$ Université de Lyon, Institut français des sciences et technologies des transports, de l'aménagement et des réseaux, Institut national de Veille Sanitaire, Unité Mixte de Recherche épidémiologique et de Surveillance Transports Travail Environnement, Lyon, France

${ }^{7}$ McLaughlin Centre for Population Health Risk Assessment, University of Ottawa, Ottawa, Canada 
${ }^{8} \mathrm{BC}$ Cancer Research Centre, BC Cancer Agency, Vancouver, Canada ${ }^{9}$ INRS-Institut Armand-Frappier, Université du Québec, Laval, Canada ${ }^{10}$ Cancer and Radiation Epidemiology Unit, Gertner Institute, Chaim Sheba Medical Center, Tel-Hashomer, Israel

${ }^{11}$ Sackler School of Medicine, Tel-Aviv University, Tel-Aviv, Israel

${ }^{12}$ School of Population Health, University of Auckland, Auckland, New Zealand

${ }^{13}$ Department of Diagnostic Imaging, Chaim Sheba Medical Center, Tel Hashomer, Israel

${ }^{14}$ Department of Population health, Hospital Research Centre (CRCHUM) University of Montreal, Montreal, Canada

\section{Acknowledgements The authors acknowledge}

- The important work of N. Varsier and J Wiart (France Telecom RD, France), M Taki (Tokyo Metropolitan University and National Institute of Information and Communications Technology, Japan), K. Wake (National Institute of Information and Communications Technology, Japan), I Deltour (Danish Cancer Centre, Copenhagen). S. Mann (Health Protection Agency, Japan), L. Ardoino (Italy) and P. Vecchia (National Institute of Health, Italy) in the development of the RF dose algorithm;

- The outstanding contribution of the neuroradiologists: Ken Faulder (Australia), William Miller, Genevieve Matte and Rafael Glickstein (Canada), Marc Hermier (Lyon) and Jacques Doyon (France) (who participated in the review of cases and also in the development and testing of the localisation protocol); Chen Hoffman (Israel) and Glen Thomson (Auckland, New Zealand) made in reviewing images and records and recording the location and origins of brain tumours in the Gridmaster;

- The collaboration of all the clinicians in the five countries who permitted access to their patients;

- The excellent work of Monika Moissonnier, Hélène Tardy and Emilie Combarlot (IARC, Lyon) in the design and validation of the international database, as well as, for MM, precious assistance in the development and testing of the RF algorithm.

In addition, the authors thank Professor Jack Siemiatycki for allowing the use of Montreal data in these analyses. The Australian team would like to acknowledge too the overall support given to study design and implementation by Associate Professor Michael Besser and Professor Andrew Kaye; and to thank fieldwork staff in Melbourne-Monique Kilkenny, Georgina Marr, Tracey McPhail, Fiona Phillips, Hayley Shaw, Yvonne Torn-Broers; and Sydney-Matthew Carroll, Sally Dunlop, Virginia MacDonald and Elizabeth Willows - the many interviewers for their hard work, and the NSW and Victorian Cancer Registries for aiding case identification. We also thank Angus Cook (University of Western Australia) and Neil Pearce (London School of Hygiene and Tropical Medicine) who, together with AW, were responsible for the conduct of the Interphone Study in New Zealand. The Canada-Ottawa centre gratefully acknowledges the work of Lynn Pratt and Daniel Bédard for their leading roles in study coordination. The Canada-Montreal team acknowledges the diligent work of fieldwork staff including Marie-Claire Goulet, Sylvie Plante, Sally Campbell and the interviewer team. The following hospitals and physicians in Montreal permitted access to their patients: CHUM-Hôpital Notre-Dame (Dr Wieslaw Michel Bojanowski, Dr Jean Jacques Dufour, Dr François Lavigne, Dr Robert A Moumdjian); Neurological Institute of Montreal (Dr Rolando Del Maestro, Dr Richard Leblanc): Hôpital du Sacré-Coeur de Montréal (Dr Marc F Giroux); The Jewish General Hospital (Dr Gerard Mohr, Dr Jamie Miles Rappaport). The Canada-Vancouver centre wishes to acknowledge the work carried out by Dr Alison Pope, Patricia Nelson, Nelson Ha, Dr Kaushik Bhagat and the interviewer team. The French team to thank the French fieldwork team, Mary-Pierre Herrscher, Fatima Lamri, Agnès Boidart, Hélène Gire, Juliette Krassilchik, Judith Lenti, Delphine Maillac, Frédérique Sonnet, Flore Taguiev, Julie Frantz, France Castay, Florian Gay, for their excellent work. The Israeli team acknowledge the diligent work of the fieldwork and office staff including Etti Aviezer, Tehila Ben-Tal, Meirav Dolev, Yonit Deutch, Tamara Rodkin, Ahuva Zultan and the interviewer team.

Funding Funding for the Interphone Study was provided by the European Fifth Framework Program, 'Quality of Life and Management of Living Resources' (contract OLK4-CT-1999901563), the International Union against Cancer (UICC). The UICC received funds for this purpose from the Mobile Manufacturers' Forum and GSM Association. Provision of funds to the Interphone Study investigators via the UICC was governed by agreements that guaranteed Interphone's complete scientific independence. The terms of these agreements are publicly available at http://www. iarc.fr/en/research-groups/RAD/RCAd.html Specific additional funds were provided for the development and analysis of the radio frequency exposure gradient and by the Fondation Santé et Radiofréquences, France and the Bundesamt fuer Strahlenschutz, Germany. The Australian centre was supported by the National Health and Medical Research Council (EME grant 219129); BKA was supported by the University of Sydney Medical Foundation Program Grant and Julianne Brown by an Australian Postgraduate Award. The Cancer Council NSW and the Cancer Council Victoria provided most of the infrastructure for the project in Australia. The Canada-Montréal data collection was funded by a grant from the Canadian Institutes of Health Research (project MOP-42525). Additionally, Dr Siemiatycki's research team was partly funded by the Canada Research Chair programme and by the Guzzo-CRS Chair in Environment and Cancer. Dr. Parent had salary support from the Fonds de recherche en santé du Québec. The other Canadian centres were supported by a university-industry partnership grant from the Canadian Institutes of Health Research (CIHR), the latter including partial support from the Canadian Wireless Telecommunications Association. The CIHR university-industry partnerships program also includes provisions that ensure complete scientific independence of the investigators. DK is the NSERC/SSHRC/McLaughlin Chair in Population Health Risk Assessment at the University of Ottawa. Additional funding for the study in France was provided by I'Association pour la Recherche sur le Cancer (ARC) (contract 5142) and three network operators (Orange, SFR, Bouygues Télécom). The funds provided by the operators represented $5 \%$ of the total cost of the French study and were governed by contracts guaranteeing the complete scientific independence of the investigators. In New Zealand, funding was provided by the Health Research Council, Hawkes Bay Medical Research Foundation and the Cancer Society of New Zealand. The findings and conclusions in this paper have not been formally disseminated by the National Institute for Occupational Safety and Health and should not be construed to represent any agency determination or policy.

Competing interests BKA's travel expenses to give two invited lecture were paid by the Australian Centre for Radio frequency Bioeffects Research, which identifies Telstra Australia as a participating institution.

Patient consent Consent was obtained from patient or next of kin if patient was too ill or deceased and next of kin responded as a proxy.

Ethics approval This study was conducted with the approval of the ethical committee of the International Agency for Research on Cancer (IARC) and appropriate local and national Institutional Review Boards (IRBs).

Contributors All authors participated in the conception and design, analysis and interpretation of data, the drafting of the article or its critical revision for important intellectual content and approved the version to be published.

Provenance and peer review Not commissioned; externally peer reviewed.

\section{REFERENCES}

1. Cardis E, Richardson L, Deltour I, et al. The INTERPHONE study: design, epidemiological methods, and description of the study population. Eur J Epidemiol 2007;22:647-64

2. Dreyer NA, Loughlin JE, Rothman KJ. Cause-specific mortality in cellular telephone users. JAMA 1999;282:1814-16.

3. Johansen C, Boice $\mathrm{J} \mathrm{Jr}$, McLaughlin J, et al. Cellular telephones and cancer-a nationwide cohort study in Denmark. J Natl Cancer Inst 2001:93:203-7.

4. Schüz J, Jacobsen $\mathrm{R}$, Olsen $\mathbf{J H}$, et al. Cellular telephone use and cancer risk: update of a nationwide Danish cohort. J Natl Cancer Inst 2006:98:1707-13.

5. Auvinen A, Hietanen M, Luukkonen R, et al. Brain tumors and salivary gland cancers among cellular telephone users. Epidemiology 2002;13:356-9.

6. Christensen HC, Schüz J, Kosteljanetz M, et al. Cellular telephones and risk for brain tumors: a population-based, incident case-control study. Neurology 2005;64:1189-95.

7. Hardell L, Näsman A, Påhlson A, et al. Use of cellular telephones and the risk for brain tumours: A case-control study. Int J Oncol 1999;15:113-16.

8. Hardell L, Carlberg M, Hansson Mild K. Pooled analysis of two case-control studies on the use of cellular and cordless telephones and the risk of benign brain tumours diagnosed during 1997-2003. Int J Oncol 2006;28:509-18.

9. Hardell L, Carlberg M, Hansson Mild K. Pooled analysis of two case-control studies on use of cellular and cordless telephones and the risk for malignant brain tumours diagnosed in 1997-2003. Int Arch Occup Environ Health 2006:79:630-9.

10. Hardell L, Mild KH, Carlberg M, et al. Tumour risk associated with use of cellular telephones or cordless desktop telephones. World J Surg Oncol 2006;4:74.

11. Hepworth SJ, Schoemaker MJ, Muir KR, et al. Mobile phone use and risk of glioma in adults: case-control study. BMJ 2006;332:883-7.

12. Hours M, Bernard M, Montestrucq $L$, et al. [Cell Phones and Risk of brain and acoustic nerve tumours: the French INTERPHONE case-control study] (In French). Rev Epidemiol Santé Publique 2007;55:321-32.

13. Inskip PD, Tarone RE, Hatch EE, et al. Cellular-telephone use and brain tumors N Engl J Med 2001:344:79-86.

14. Lahkola A, Auvinen A, Raitanen J, et al. Mobile phone use and risk of glioma in 5 North European countries. Int J Cancer 2007:120:1769-75.

15. Lahkola A, Salminen T, Raitanen J, et al. Meningioma and mobile phone use-a collaborative case-control study in five North European countries. Int J Epidemiol 2008:37:1304-13.

16. Lönn S, Ahlbom A, Hall $P$, et al. Long-term mobile phone use and brain tumor risk Am J Epidemiol 2005:161:526-35

17. Muscat JE, Malkin MG, Thompson S, et al. Handheld cellular telephone use and risk of brain cancer. JAMA 2000;284:3001-7.

18. Schuz J, Bohler E, Berg G, et al. Cellular phones, cordless phones, and the risks of glioma and meningioma (Interphone Study Group, Germany). Am J Epidemiol 2006;163:512-20. 
19. Klaeboe L, Blaasaas KG, Tynes T. Use of mobile phones in Norway and risk of intracranial tumours. Eur J Cancer Prev 2007;16:158-64

20. Takebayashi T, Varsier N, Kikuchi Y, et al. Mobile phone use, exposure to radiofrequency electromagnetic field, and brain tumour: a case-control study. $\mathrm{Br} J$ Cancer 2008;98:652-9.

21. Kainz W, Christ A, Kellom T, et al. Dosimetric comparison of the specific anthropomorphic mannequin (SAM) to 14 anatomical head models using a novel definition for the mobile phone positioning. Phys Med Biol 2005:50:3423-45

22. Wiart J, Mittra R, Chaillou $S$, et al. The analysis of human head interaction with a hand-held mobile using the non-uniform FDTD, In Proceedings IEEE AP-S Wireless Commun. Conf., Nov. 1998, pp. 77-80.

23. Cardis E, Deltour I, Mann S, et al. Distribution of RF energy emitted by mobile phones in anatomical structures of the brain. Phys Med Biol 2008;53:2771-83
24. Cardis E, Varsier N, Bowman JD, et al. Estimation of RF energy absorbed in the brain from mobile phones in the Interphone study. Occup Environ Med 2011:68:686-93.

25. Cardis E, Kilkenny M. International Case-Control Study of Cancers of Brain and Salivary Gland-Report of the Feasibility Study. 99/004. Lyon: International Agency for Research on Cancer (IARC), 1999. IARC Internal Reports.

26. GridMaster Computer Program. Düsseldorf: Vompras, 2007.

27. Vrijheid $\mathbf{M}$, Cardis $\mathrm{E}$, Varsier $\mathrm{N}$, et al. Tumour localisation in the Interphone international study of mobile phone radiofrequency exposure and brain tumour risk. (in preparation).

28. The Interphone Study Group. Brain tumour risk in relation to mobile telephone use: results of the INTERPHONE international case-control study. Int J Epidemiol 2010;39:675-94

29. Vrijheid M, Armstrong BK, Bédard D, et al. Recall bias in the assessment of exposure to mobile phones. J Expo Sci Environ Epidemiol 2009:19:369-81.

\section{APPENDIX}

Table Al Distributions of glioma and meningioma cases and controls ascertained, interviewed and matched and with data available for analysis

\begin{tabular}{|c|c|c|c|c|c|c|c|}
\hline & \multirow[b]{2}{*}{$\begin{array}{l}\text { Total } \\
\text { ascertained }\end{array}$} & \multirow[b]{2}{*}{ Interviewed } & \multirow[b]{2}{*}{$\begin{array}{l}\text { Matched to case or } \\
\text { controls interviewed } \\
\text { within } 1 \text { year }\end{array}$} & \multicolumn{4}{|c|}{ Cases with tumour localisation data and their matched controls } \\
\hline & & & & $\begin{array}{l}\text { Centre estimated by } \\
\text { neuroradiologist or } \\
\text { computer algorithm }\end{array}$ & $\begin{array}{l}\text { As in preceding column } \\
\text { and have information about } \\
\text { communication system } \\
\text { and frequency band }\end{array}$ & $\begin{array}{l}\text { Centre estimated } \\
\text { by neuroradiologist }\end{array}$ & $\begin{array}{l}\text { As in preceding column } \\
\text { and have information about } \\
\text { communication system } \\
\text { and frequency band }\end{array}$ \\
\hline \multicolumn{8}{|l|}{ Cases } \\
\hline Total & 1302 & 829 & 809 & 567 & 553 & 339 & 329 \\
\hline Australia & 536 & 301 & 295 & 142 & 142 & 141 & 141 \\
\hline Canada & 273 & 170 & 170 & 158 & 144 & 110 & 100 \\
\hline France & 155 & 94 & 94 & 68 & 68 & 56 & 56 \\
\hline Israel & 206 & 180 & 168 & 163 & 163 & 0 & 0 \\
\hline New Zealand & 132 & 84 & 82 & 36 & 36 & 32 & 32 \\
\hline \multicolumn{8}{|l|}{ Controls } \\
\hline Total & 4838 & 2565 & 2496 & 1877 & 1762 & 1032 & 958 \\
\hline Australia & 1608 & 669 & 669 & 328 & 317 & 327 & 316 \\
\hline Canada & 1330 & 653 & 633 & 581 & 492 & 348 & 296 \\
\hline France & 639 & 472 & 459 & 331 & 328 & 294 & 291 \\
\hline Israel & 911 & 599 & 587 & 566 & 562 & 0 & 0 \\
\hline New Zealand & 350 & 172 & 148 & 71 & 63 & 63 & 55 \\
\hline \multicolumn{8}{|l|}{ Meningioma } \\
\hline & & & & \multicolumn{4}{|c|}{ Cases with tumour localisation data and their matched controls } \\
\hline & $\begin{array}{l}\text { Total } \\
\text { ascertained }\end{array}$ & Interviewed & $\begin{array}{l}\text { Matched to controls } \\
\text { or case interviewed } \\
\text { within } 1 \text { year }\end{array}$ & $\begin{array}{l}\text { Tumour centre } \\
\text { estimated by } \\
\text { neuroradiologist or } \\
\text { computer algorithm }\end{array}$ & $\begin{array}{l}\text { As in preceding column } \\
\text { and have information about } \\
\text { communication system } \\
\text { and frequency band }\end{array}$ & $\begin{array}{l}\text { Tumour centre } \\
\text { estimated by } \\
\text { neuroradiologist }\end{array}$ & $\begin{array}{l}\text { As in preceding column } \\
\text { and have information about } \\
\text { communication system } \\
\text { and frequency band }\end{array}$ \\
\hline \multicolumn{8}{|l|}{ Cases } \\
\hline Total & 1199 & 895 & 842 & 688 & 676 & 349 & 343 \\
\hline Australia & 413 & 254 & 250 & 158 & 158 & 155 & 155 \\
\hline Canada & 134 & 94 & 94 & 82 & 71 & 56 & 50 \\
\hline France & 190 & 145 & 144 & 116 & 116 & 109 & 109 \\
\hline Israel & 390 & 350 & 306 & 303 & 302 & 0 & 0 \\
\hline New Zealand & 72 & 52 & 48 & 29 & 29 & 29 & 29 \\
\hline \multicolumn{8}{|l|}{ Controls } \\
\hline Total & 4838 & 2565 & 2464 & 2015 & 1911 & 1213 & 1142 \\
\hline Australia & 1608 & 669 & 667 & 431 & 413 & 417 & 400 \\
\hline Canada & 1330 & 653 & 613 & 540 & 466 & 342 & 295 \\
\hline France & 639 & 472 & 458 & 379 & 376 & 358 & 356 \\
\hline Israel & 911 & 599 & 591 & 569 & 565 & 0 & 0 \\
\hline New Zealand & 350 & 172 & 135 & 96 & 91 & 96 & 91 \\
\hline
\end{tabular}


Table All ORs for brain tumours with level of total cumulative specific RF energy (TCSE) exposure (in joules per kilogram) in subjects with tumour centre estimated by a neuroradiologist or computer algorithm, excluding non-regular users. $n$ these analyses, the lowest quintile of TCSE is used as the reference category

\begin{tabular}{llll}
\hline & \multicolumn{3}{l}{$\begin{array}{l}\text { Subjects with tumour centre estimated by } \\
\text { a neuroradiologist or computer algorithm }\end{array}$} \\
\cline { 2 - 4 } & Cases & Controls & OR (95\% CI) \\
\hline Glioma & & \\
$<76.7$ & 65 & 241 & 1.00 \\
$76.7-$ & 68 & 213 & 1.34 (0.86 to 2.08) \\
$284.1-$ & 60 & 198 & $1.13(0.71$ to 1.79$)$ \\
$978.9-$ & 57 & 191 & $1.22(0.77$ to 1.93$)$ \\
$3123.9+$ & 103 & 195 & $1.93(1.26$ to 2.96$)$ \\
Meningioma & & & \\
$<76.7$ & 96 & 231 & 1.00 \\
$76.7-$ & 71 & 194 & $0.86(0.58$ to 1.27$)$ \\
$284.1-$ & 56 & 221 & $0.63(0.41$ to 0.95$)$ \\
$978.9-$ & 62 & 199 & $0.87(0.58$ to 1.31$)$ \\
$3123.9+$ & 88 & 237 & $1.04(0.71$ to 1.53$)$ \\
\hline
\end{tabular}

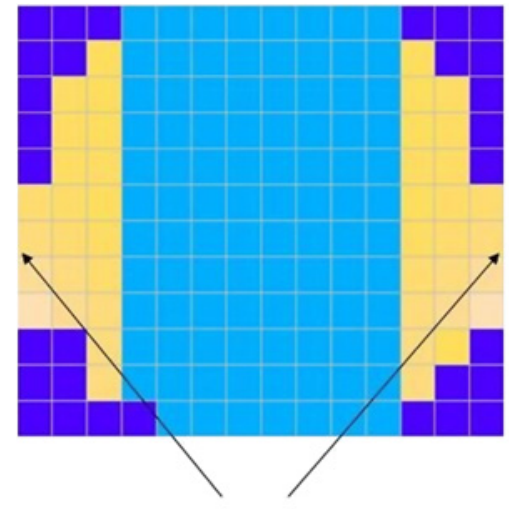

Coronal view of the brain

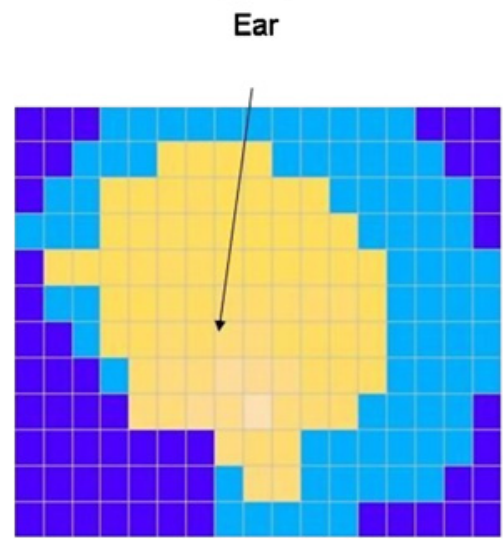

Sagittal view of the brain

Figure Al Spatial deposition of radio frequency (RF) energy in the Gridmaster cells-the light cubes identify the most exposed region of the brain, that is, that with the highest specific absorption rate of RF energy from mobile phones. The dark cubes identify the less exposed area of the brain. The most exposed area (light cubes) correspond to about $16 \%$ of the brain volume and absorbs $>50 \%$ of the total RF energy in the brain from mobile phones used at the ear.
Figure All Distribution of glioma cases and controls by level of total cumulative specific energy (TCSE) (in joules per kilogram) at the estimated centre of the tumour; centre is taken to be as estimated by neuroradiologist, where available, and as estimated by computer algorithm otherwise.
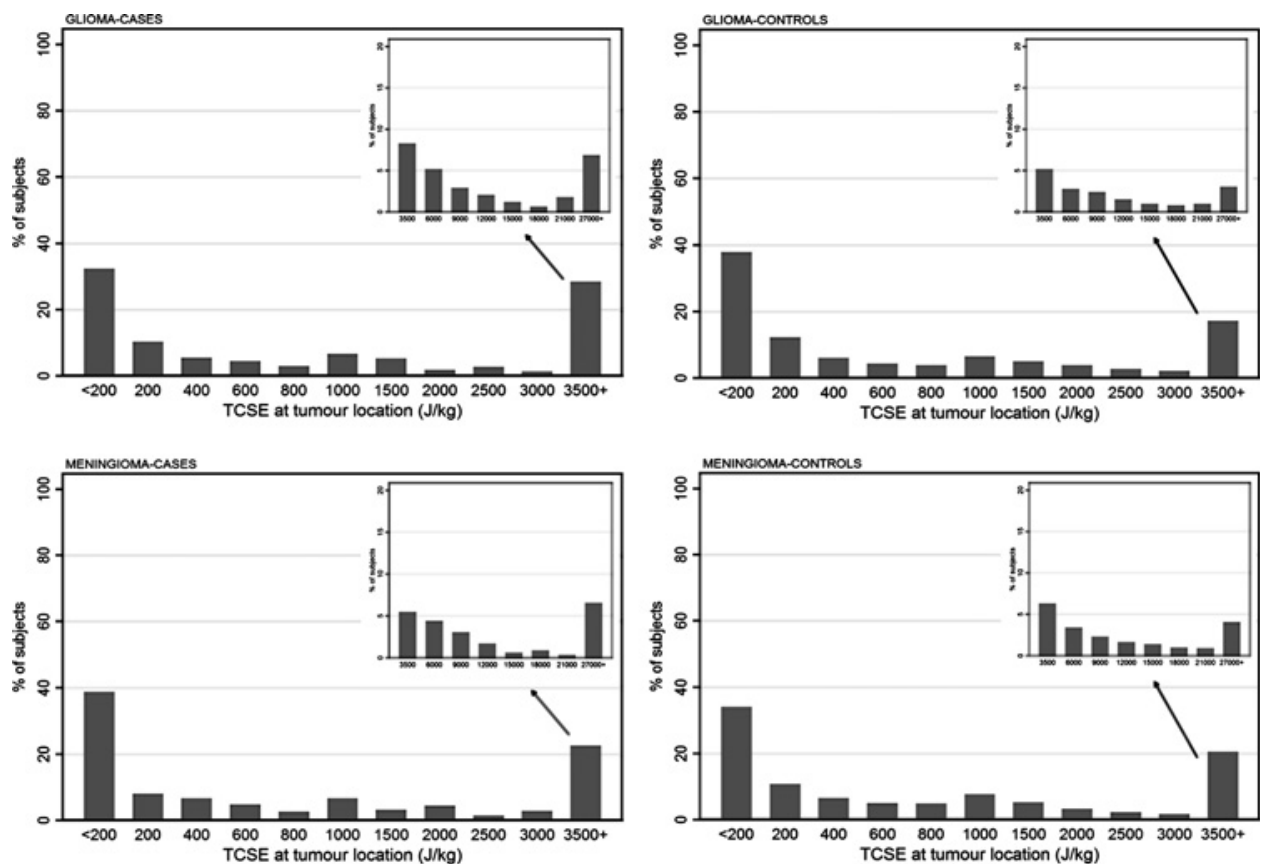